\title{
Prognostic importance of some clinical and therapeutic factors for the effect of portal vein embolization in patients with primarily inoperable colorectal liver metastases
}

\author{
Vladislav Treskaํ, Tomas Skalicky¹, Alan Sutnar ${ }^{1}$, Liska Vaclav¹, Jakub Fichtl'1, Judita Kinkorova², \\ Monika Vachtova ${ }^{1}$, Andrea Narsanska ${ }^{1}$
}

${ }^{1}$ University Hospital, School of Medicine, Pilsen, Czech Republic

${ }^{2}$ Academy of Science, Prague, Czech Republic

Submitted: 15 February 2011

Accepted: 18 August 2011

Arch Med Sci 2013; 9, 1: 47-54

DOI: 10.5114/aoms.2013.33348

Copyright @ 2013 Termedia \& Banach

\author{
Corresponding author: \\ Prof. Vladislav Treska \\ Department of Surgery \\ University Hospital \\ Alej Svobody 80 \\ 30460 Pilsen, Czech Republic \\ Phone: +420377 104270 \\ Fax: +42 0377103965 \\ E-mail: treska@fnplzen.cz
}

\begin{abstract}
Introduction: Portal vein embolization (PVE) may increase the resectability of liver metastases. However, the problem of PVE is insufficient growth of the liver or tumor progression in some patients. The aim of this study was to evaluate the significance of commonly available clinical factors for the result of PVE. Material and methods: Portal vein embolization was performed in 38 patients with colorectal liver metastases. Effects of age, gender, time between PVE and liver resection, oncological therapy after PVE, indocyanine green retention rate test, synchronous, metachronous and extrahepatic metastases, liver volume before and after PVE, increase of liver volume after PVE and the quality of liver parenchyma before PVE on the result of PVE were evaluated.

Results: Liver resection was performed in $23(62.2 \%)$ patients within $1.3 \pm 0.4$ months after PVE. Tumor progression occurred in 9 (23.7\%) patients and $6(15.8 \%)$ patients had insufficient liver hypertrophy. Significant clinical factors of PVE failure were number of liver metastases (cut-off - 4; odds ratio - 4.7; $p<0.03$ ), liver volume after PVE (cut-off $1000 \mathrm{~cm}^{3}$; odds ratio - 5.1; $p<0.02$ ), growth of liver volume after PVE (cut-off $150 \mathrm{~cm}^{3}$; odds ratio $-18.7 ; p<0.002$ ), oncological therapy administered concomitantly with PVE $(p<0.003)$.

Conclusions: Negative clinical factors of resectability of colorectal cancer liver metastases after PVE included more than four liver metastases, liver volume after PVE $<1000 \mathrm{~cm}^{3}$, growth of the contralateral lobe by less than $150 \mathrm{~cm}^{3}$ and concurrent oncological therapy.
\end{abstract}

Key words: colorectal liver metastases, portal vein embolization, prognostic factors.

\section{Introduction}

Currently, the resectability of colorectal liver metastases (CLM) is unfortunately still low (20-25\%). One of the causes is insufficient future liver remnant volume (FLRV) after liver resection to ensure liver function. Portal vein embolization (PVE) is a method which significantly extends resectability of primarily inoperable CLM. Portal vein embolization induces atrophy of the embolized liver lobe and contralateral lobe compensatory hypertrophy. It is indicated if FLRV is less than $30 \%$ in patients with normal liver and less than $40 \%$ in cirrhotic liver $[1,2]$. Unfortunately, due to unknown reasons sufficient liver hypertrophy does not develop in some patients. Portal vein embolization not only can stimulate the growth of 
FLRV but also can affect tumor growth. Several authors have reported tumor proliferation in the liver or in the body after PVE [3-5].

Therefore, the purpose of our study was to find out whether the result of PVE in patients with CLM can be predicted based on commonly available clinical parameters.

\section{Material and methods}

Between 2000 and 2010, we operated in total on 347 patients with CLM. The FLRV was insufficient in 38 patients and we performed PVE on the side of the tumor. We performed PVE in the first patients using embolization coils, and currently we use the embolization mixture Histoacryl (B. Braun, Melsungen, Germany): Lipiodol (Cedex, Rennes, France) diluted in the ratio of $1: 10$. If needed, we added the absorbable gelatin mass Spongostan (Johnson \& Johnson, New Brunswick, USA) to the embolization mixture. The mean age of patients was $60.8 \pm 7.6$ years (33.3-70.6 years), 26 males and 12 females. Each patient signed the informed consent before PVE. Fifty-six percent of the patients had > 4 CLM. Synchronous, metachronous and extrahepatic metastases occurred in 21 (55.3\%), $17(44.7 \%)$ and $6(15.8 \%)$ patients, respectively (Table I). Extrahepatic metastases were not a contraindication for PVE provided that they were radically removable at one time, or in stages. We performed the functional test using the liver clearance of indocyanine green (ICG) in the Limon device (Pulsion Medical, Systems, Munich, Germany) in all patients before PVE. If the test result was higher than $14 \%$ (in 3 patients) the test was repeated after the successful PVE before liver resection to evaluate the extent of the surgery that we selected on the basis of the volumetric and functional criteria listed in Table I. We evaluated the quality of liver parenchyma using the liver biopsy based on criteria according to Ishak and Bruntova (Tables II, III). We also evaluated the number of CLM and liver tissue volume (in $\mathrm{cm}^{3}$ ) before and after the PVE together with the extrahepatic metastases in the Somatom Definition (Siemens, Forchheim, Germany) device using the Syngo Volume Calculation software of the same company. We performed chemotherapy together with PVE in 9 (23.7\%) patients using the FOLFOX 4 regimen (oxaliplatin $85 \mathrm{mg} / \mathrm{m}^{2}$, leucovorin $200 \mathrm{mg} / \mathrm{m}^{2}$ intravenously over $120 \mathrm{~min}$, followed by 5 -fluorouracil $400 \mathrm{mg} / \mathrm{m}^{2}$ as intravenous bolus over 2-4 min, followed by 5 -fluorouracil $600 \mathrm{mg} / \mathrm{m}^{2}$ in a 22-hour intravenous infusion) [6] with administration of bevacizumab $5 \mathrm{mg} / \mathrm{kg}$ intravenously and the whole cycle was repeated after 2 weeks [7].

\section{Statistical analysis}

Basic statistical data, such as mean, standard deviation, variance, median, interquartile range, minimum and maximum for the measured parameters in the whole group and in the single groups and subgroups were calculated. The frequencies were calculated for the categorical parameters. Nonparametric tests, such as the Kruskal-Wallis and Wilcoxon test, were used to compare the distribution of the single parameters in different groups and subgroups because of the distribution of these variables. The categorical variables were analyzed using the $\chi^{2}$ test and the clinical significance of these variables was investigated by odds ratio. Statistical analysis was performed using the Statistica 9.0 software.

\section{Results}

Technically, PVE in all patients was sufficient with complete elimination of the portal venous flow in the embolized liver lobe. The mortality and morbidity of patients in the form of subcapsular liver hematoma in one patient after the performed PVE was $0 \%$ and $2.6 \%$, respectively (subcapsular liver hematoma in 1 patient). Hypertrophy of the contralateral liver lobe after PVE occurred in 23 (62.2\%) patients on average within $1.3 \pm 0.4$ months with subsequent radical (RO) liver resection. Tumor progression in the liver or in the body occurred in $9(23.7 \%)$ patients. Insufficient hypertrophy of the liver parenchyma after PVE occurred in 6 (15.8\%) patients (Table IV).

Important clinical factors which affect the result of PVE included the number of CLM, liver volume after PVE, increase of liver volume after PVE, and chemotherapy with biological therapy performed simultaneously with PVE. If patients had more than 4 CLM before PVE, then the probability of failure of PVE (tumor progression, insufficient liver hypertrophy) would be high $(p<0.03)$. If the liver volume after PVE was $<1000 \mathrm{~cm}^{3}$ the patients had

Table I. Functional and volumetric criteria of the liver resections

\begin{tabular}{|lccc|}
\hline Parameter & $\begin{array}{c}\text { Large liver resection } \\
(\geq 3 \text { liver segments })\end{array}$ & $\begin{array}{c}\text { Small liver resection } \\
(<3 \text { liver segments })\end{array}$ & $\begin{array}{c}\text { Contraindication } \\
\text { of liver resection }\end{array}$ \\
\hline ICG test (15 min) [\%] & $<14$ & $14-20$ & $>20$ \\
\hline FLRV - CT volumetry - normal parenchyma [\%] & $>40$ & $25-40$ & $<25$ \\
\hline FLRV - CT volumetry - cirrhosis [\%] & $>70$ & $50-70$ & $<50$ \\
\hline
\end{tabular}


Table II. Evaluation of liver parenchyma according to Ishak

\begin{tabular}{|c|c|}
\hline \multicolumn{2}{|l|}{ GRADING } \\
\hline \multicolumn{2}{|l|}{ A. Interface hepatitis (piecemeal necroses) } \\
\hline None & 0 \\
\hline Mild (focal, in few portal fields) & 1 \\
\hline Mild to moderate (focal, in most of the fields) & 2 \\
\hline Moderate (continual, affecting less than $50 \%$ of field peripheries and septa) & 3 \\
\hline Severe (continual, affecting more than $50 \%$ of field peripheries and septa) & 4 \\
\hline \multicolumn{2}{|l|}{ B. Confluent necroses } \\
\hline None & 0 \\
\hline Focal & 1 \\
\hline Zonal in several zones 3 & 2 \\
\hline Zonal in most of zone 3 & 3 \\
\hline Zonal in zone 3 and infrequent porto-central necroses & 4 \\
\hline Zonal in zone 3 and multiple porto-central necroses & 5 \\
\hline Multi-acinar and/or pan-acinar necroses & 6 \\
\hline \multicolumn{2}{|l|}{ C. Focal lytic necroses and apoptosis } \\
\hline None & 0 \\
\hline $0-1 /$ field of view with the objective of $10 x$ & 1 \\
\hline 2-4/field of view with the objective of $10 x$ & 2 \\
\hline $5-10 /$ field of view with the objective of $10 x$ & 3 \\
\hline$>10 /$ field of view with the objective of $10 x$ & 4 \\
\hline \multicolumn{2}{|l|}{ D. Portal inflammatory infiltration } \\
\hline None & 0 \\
\hline Mild & 1 \\
\hline Moderate & 2 \\
\hline Moderate to severe in all portal fields & 3 \\
\hline Severe in all portal fields & 4 \\
\hline \multicolumn{2}{|l|}{ STAGING } \\
\hline No fibrosis & 0 \\
\hline Fibrous extension of some portal fields with or without short fibrous septa & 1 \\
\hline Fibrous extension of most portal fields with or without short fibrous septa & 2 \\
\hline Fibrous extension of most portal fields with infrequent porto-portal septa & 3 \\
\hline Fibrous extension of the portal fields with frequent porto-portal and porto-central septa & 4 \\
\hline Frequent porto-portal and/or porto-central septa with infrequent nodules (imminent cirrhosis) & 5 \\
\hline Cirrhosis & 6 \\
\hline
\end{tabular}

a 5.1 times higher probability of failure of PVE compared to those who experienced an increase in liver parenchyma of $>1000 \mathrm{~cm}^{3}$ following PVE $(p<0.02)$. If the increase of liver volume after PVE was less than $150 \mathrm{~cm}^{3}$, then the risk of unsuccessful PVE was 18.7 times higher $(p<0.002)$ - Table IV. The liver volume before PVE was not statistically significant $(p<0.06)$ with regard to the result of PVE (Table V). In $91 \%$ of patients in the group with liver hypertrophy after PVE no concomitant oncological therapy (FOLFOX 4 + bevacizumab) was performed. In the group of patients with tumor progression after PVE no concomitant oncological treatment was administered in $82 \%$ of these patients. However, concomitant oncological therapy was performed in $71 \%$ of patients in the group in which no increase of the liver parenchyma occurred after PVE $(p<0.03)$ - Table VI. No effect on the result of PVE was found for other monitored clinical factors Table VII. 
Table III. Evaluation of liver parenchyma according to Bruntova

\begin{tabular}{|l|}
\hline \multicolumn{1}{|c|}{ GRADING } \\
\hline $\begin{array}{l}\text { Steatosis up to 66\% of the surface, balloon degeneration of infrequent hepatocytes in zone 3, } \\
\text { infrequent disperse neutrophils and lymphocytes in the lobules, no or minimal portal inflammation }\end{array}$ \\
\hline $\begin{array}{l}\text { Steatosis of any grade, balloon degeneration of numerous hepatocytes (mainly in zone 3), well apparent intralobular } \\
\text { neutrophils, pericellular fibrosis in zone 3, mild to moderate portal and lobular chronic inflammatory infiltration }\end{array}$ \\
\hline $\begin{array}{l}\text { Pan-acinar steatosis, significant balloon degeneration mainly in zone 3 associated with numerous lobular } \\
\text { neutrophils, mild to moderate portal and lobular inflammatory infiltration }\end{array}$ \\
\hline STAGING \\
\hline Perisinusoidal/pericellular fibrosis in zone 3 3 \\
\hline Perisinusoidal/pericellular fibrosis in zone 3 and periportal fibrosis with porto-portal and/or porto-central septa \\
\hline Cirrhosis 3
\end{tabular}

Table IV. Number of CLM, liver volume after PVE, liver volume change after PVE

\begin{tabular}{|lcccc|}
\hline Parameter & Cut-off & $95 \%$ Cl OR & OR & Value of $p$ \\
\hline Number of CLM & 4 & $1.2-19.2$ & 4.7 & $<0.03$ \\
\hline Liver volume after PVE $\left[\mathrm{cm}^{3}\right]$ & 1000 & $1.3-20.5$ & 5.1 & $<0.02$ \\
\hline Liver volume change after PVE $\left[\mathrm{cm}^{3}\right]$ & 150 & $2.0-171.9$ & 18.7 & $<0.002$ \\
\hline
\end{tabular}

Table V. Liver volume before and after PVE, liver volume changes after PVE - results of PVE

\begin{tabular}{|c|c|c|c|c|c|c|c|}
\hline Parameter & $\begin{array}{c}\text { Liver } \\
\text { hypertrophy }\end{array}$ & $\begin{array}{c}\text { Tumor } \\
\text { progression }\end{array}$ & iLH & $\begin{array}{l}\text { ANOVA } \\
(p)\end{array}$ & Success & Failure & $\begin{array}{c}\text { ANOVA } \\
(p)\end{array}$ \\
\hline Liver volume before PVE [ $\left.\mathrm{cm}^{3}\right]$ & $476.0 \pm 134.1$ & $415.5 \pm 117.9$ & $350.6 \pm 82.1$ & $<0.06$ & $476.0 \pm 134.1$ & $388.8 \pm 106.9$ & $<0.06$ \\
\hline Liver volume after PVE [ $\left.\mathrm{cm}^{3}\right]$ & $1022.9 \pm 273.9$ & $858.8 \pm 374.5$ & $629.0 \pm 250.1$ & $<0.01$ & $1022.9 \pm 273.9$ & $769.4 \pm 343.3$ & $<0.02$ \\
\hline Liver volume change $\left[\mathrm{cm}^{3}\right]$ & $546.8 \pm 331.7$ & $403.0 \pm 380.5$ & $278.4 \pm 216.8$ & $<0.08$ & $546.8 \pm 331.7$ & $351.7 \pm 321.0$ & $<0.04$ \\
\hline
\end{tabular}

Table VI. Chemotherapy, biological therapy and the result of PVE

\begin{tabular}{|c|c|c|c|c|c|c|c|}
\hline CHBT & $\begin{array}{l}\text { Liver } \\
\text { hypertrophy [\%] }\end{array}$ & $\begin{array}{c}\text { Tumor } \\
\text { progression [\%] }\end{array}$ & iLH [\%] & Value of $p$ & $\begin{array}{c}\text { Success } \\
{[\%]}\end{array}$ & $\begin{array}{c}\text { Failure } \\
\text { [\%] }\end{array}$ & Value of $p$ \\
\hline Yes & 9.1 & 18.2 & 71.4 & \multirow[t]{2}{*}{$<0.003$} & 9.1 & 38.9 & \multirow[t]{2}{*}{$<0.03$} \\
\hline No & 90.9 & 81.8 & 28.6 & & 90.9 & 61.1 & \\
\hline
\end{tabular}

CHBT - chemotherapy, biological therapy, iLH - insufficient liver hypertrophy, Success - liver hypertrophy, Failure - iLH or tumor progression

Table VII. Factors with no effect on the result of PVE

\begin{tabular}{|c|c|c|c|c|}
\hline Factors & Liver hypertrophy & $\mathrm{iLH}$ & Tumor progression & ANOVA $(p)$ \\
\hline Age & $59.9 \pm 6.6$ & $60.9 \pm 4.8$ & $62.5 \pm 10.8$ & $<0.17$ \\
\hline Gender M/F & $13 / 9$ & $7 / 0$ & $8 / 3$ & $<0.12$ \\
\hline ICG test $[\%]$ & $3.8 \pm 3.4$ & $2.3 \pm 1.3$ & $7.8 \pm 8.9$ & $<0.84$ \\
\hline Synchronous/metachronous CLM [\%] & $61.9 / 38.1$ & $50 / 50$ & $45.4 / 54.6$ & $<0.65$ \\
\hline Extrahepatic CLM yes/no [\%] & $22.7 / 77.3$ & $14.3 / 85.7$ & $18.2 / 81.8$ & $<0.87$ \\
\hline Ishak GOSO/others [\%] & $59.1 / 40.9$ & $100 / 0$ & $100 / 0$ & $<0.29$ \\
\hline Bruntova G0S0/others [\%] & $90.0 / 9.1$ & $50 / 50$ & $100 / 0$ & $<0.19$ \\
\hline PVE interval - surgery [months] & $1.3 \pm 0.4$ & $1.6 \pm 1.2$ & $1.7 \pm 2.1$ & $<0.53$ \\
\hline
\end{tabular}

iLH-insufficient liver hypertrophy 


\section{Discussion}

Unfortunately, the Czech Republic has a leading position in the incidence of colorectal cancer in the world [8]. We can assume that this would be the same for the incidence of CLM. Radical liver resection together with oncological therapy are the only hope for patients with CLM. Unfortunately, only $20-30 \%$ of patients with CLM are able to undergo surgery at the time of diagnosis. Apart from intraor extrahepatic growth of the tumor, the reasons for non-resectability of CLM also include insufficient FLRV, which is needed to ensure the metabolic needs of the organism in the postoperative period $[9,10]$. There is a general rule that at least $30 \%$ of healthy liver parenchyma must be preserved for a safe liver resection and in patients with liver cirrhosis or liver damage of different origin (e.g. after chemotherapy, in cholestasis) at least $70-80 \%$ of liver parenchyma must be intact. If the mortality after primary large liver resections (more than 3 liver segments) in patients with functionally healthy liver ranges between 3.2 and $7 \%$, then it may reach up to $32 \%[11,12]$ in patients with liver cirrhosis or with liver impairment of different origin.

Portal vein embolization is indicated as the first step before a large liver resection in patients with insufficient FLRV. In many patients hypertrophy of the liver parenchyma occurs after PVE at intervals of 2-8 weeks by $20-46 \%$, and $70-100 \%$ of them may undergo liver resection at an interval of 4- 6 weeks after PVE [13]. However, in some patients there is insufficient increase in the volume of the contralateral liver lobe after PVE. This mainly concerns patients with chronic liver disease, diabetes, or patients in whom a recanalization of the embolized portal branch occurred after PVE, or patients presenting portal hypertension with porto-systemic shunts [14, 15]. However, the causes of insufficient liver hypertrophy are not clear in most of the patients.

Progression of the tumor in the liver or in the body after PVE is a serious problem $[16,17]$. The causes are unclear even though a number of growth factors and cytokines, which play a very significant role in regeneration of liver parenchyma after PVE, are considered in progression of tumors in the liver and body. Because of PVE associated with atrophy of the embolized liver lobe, the growth of the contralateral liver lobe is initiated due to both metabolic and hemodynamic changes in the portal and arterial hepatic circulation [18]. Fast hepatocyte replication occurs after PVE and it is followed by the growth and increase of the volume of hepatocytes after several days. The non-parenchymal cells (Kupffer cells, endothelial cells, cholangiocytes) replicate for several days following the replication of hepatocytes. The regeneration process is controlled by a number of mediators. Under normal circumstances hepatocytes are in the so-called G0 rest period. After PVE the hepatocytes in the nonembolized liver tissue enter into the G1 phase, which is stimulated by cytokines - tumor necrosis factor $\alpha$, interleukin 6 , insulin and prostaglandins. Another step of liver regeneration is the $\mathrm{S}$ phase, which is stimulated by growth factors - epidermal growth factor, transforming growth factor $\alpha$ and serotonin. Termination of liver regeneration is then regulated by another factor - transforming growth factor $\beta[19,20]$. Very important cells which probably take a significant part in hypertrophy of the liver parenchyma are the progenitor cells, which can differentiate into hepatocytes and cholangiocytes. Other important cells in the liver parenchyma include the stem cells, either hematopoietic (hematopoietic stem cells) or mesenchymal (mesenchymal stem cells). Their role consists in the fact that they can supplement the amount of progenitor cells and hence increase their proliferation activity, but at the same time they can differentiate into hepatocytes and cholangiocytes [21, 22]. The hemodynamic factor plays an important role in the process of hypertrophy of the liver parenchyma. Under physiological conditions up to $80 \%$ of blood comes to the liver from the portal vein and the remaining $20 \%$ comes from the arterial circulation. After PVE the blood flow through the portal vein in the non-embolized lobe is significantly increased, and it is compensated by an increase of the arterial flow in the embolized lobe known as the "hepatic arterial buffer response" [23, 24].

The above processes may play an important role in the progression of CLM, or in the body after PVE. Colorectal liver metastases are mainly supplied with arterial blood, and in addition to the above described factors, which play an important role in the regeneration of liver parenchyma, they will undoubtedly be involved in various degrees in the process of tumor growth $[25,26]$. Stimulation of the growth of so-called micrometastases in the liver, which are not visible with available diagnostic methods before PVE, may occur. Growth of the tumor (especially micrometastases) elsewhere in the body may be an issue, because all the above stated mediators are released into the circulation after PVE.

Because we usually have to wait for the outcome of PVE as well as for the possibility of radical resection for several weeks, in most cases 4-6 weeks, it would certainly be good to determine, using readily available clinical indicators, the patient's chances for the success of PVE, and according to this suitably modify our treatment procedures. In our study, we chose commonly available clinical parameters such as age, gender, time between PVE and liver resection, oncological treatment after PVE, the ICG test, synchronous, metachronous and extrahepatic metastases, liver volume before and after PVE, change in liver volume after PVE and the quality of liver parenchyma evaluated by Ishak and Bruntova. Inter- 
esting clinical factors with regard to the success of PVE included the number of CLM, liver volume after PVE, increase in liver volume after PVE and oncological treatment carried out together with PVE.

The number of CLM is a known risk factor of the success of liver resection. It can generally be assumed that the number of CLM will be a significant factor for the outcome of PVE. The more CLM in the embolized part of the liver, the more likely are the so-called micrometastases in the remaining liver parenchyma and the higher the risk of tumor progression after PVE because of the stimulation of their growth by growth factors and cytokines released after PVE to the circulation [27]. An increase of the proliferative activity of liver metastases was documented after PVE using the criteria of the Ki- 67 index compared with liver metastases in patients in whom PVE was not performed [28]. Similar growth activity was described in primary liver tumors [29], where tumor growth after PVE was $2.37 \mathrm{~cm}^{3} /$ day compared to $0.59 \mathrm{~cm}^{3} /$ day before PVE.

Of course, the higher the increase in liver volume after PVE, the greater the chance for radical liver resection due to CLM. However, a higher growth in liver parenchyma after PVE was also found in patients with tumor progression in the liver. The increase in volume in these patients did not reach such an intensity as in those who subsequently underwent a liver resection, but it was significantly higher than in patients in whom PVE was without any significant effect. The above-mentioned factors which cause liver hypertrophy on the one hand and tumor progression on the other will undoubtedly participate also in this case. It will be very difficult and almost impossible to determine where the limit of risk is for the final liver volume after PVE with regard to liver hypertrophy and tumor progression. However, in the meantime, we have a small number of patients with tumor progression after PVE available and our research continues in this respect. Certainly, it will be interesting to consider from this view the final volume of the liver, but especially the growth rate of liver parenchyma after PVE, because it is known that the largest growth activity of the liver parenchyma occurs in the first 14 days after PVE. The same mechanism can be expected even in progression of the CLM after PVE. If we evaluated (given the number of our patients) the effect of the change of the liver volume on the success (hypertrophy) or failure (insufficient hypertrophy, tumor progression) of PVE, then the patients with the growth of hepatic parenchyma by no more than $150 \mathrm{~cm}^{3}$ had a significantly higher risk of failure.

Similarly, the final volume of the liver after PVE was significant for the outcome of PVE. Patients whose liver volume was $>1000 \mathrm{~cm}^{3}$ had a greater chance for successful liver resection after PVE.
The question of concomitant oncological therapy after PVE is still under discussion. In the literature there has not been a common consensus on the oncological treatment in terms of interruption or continuation after the performed PVE. Some authors recommend discontinuing systemic chemotherapy in patients indicated for PVE several weeks before PVE and to resume it again 3 to 4 weeks after liver resection [30-33]. The reason is the possible impairment of the liver functions after administration of oxaliplatin (sinusoidal congestion) or irinotecan (steatosis) [34] associated with insufficient regeneration of the liver parenchyma after PVE. However, other authors support the opinion that systemic chemotherapy including fluorouracil with or without oxaliplatin, and irinotecan with bevacizumab performed together with PVE is not in most cases a setback for hypertrophy of the nonembolized lobe, and to some extent it prevents proliferation of the liver tumor or system micrometastases in the interval needed for growth of the non-embolized lobe after PVE [35]. It can also be used with benefit in cases when chemotherapeutic "down-staging" of the liver tumor $[36,37]$ is needed before the resection after PVE. In addition, the anti-angiogenic effect of bevacizumab may play an important role in the phenomenon known as "hepatic arterial buffer response" in the area of the embolized liver lobe. Nevertheless, some caution in systemic chemotherapy is needed in case of impairment of the liver structure by fibrosis or cirrhosis during PVE [38-40]. Our results do not provide an unambiguous answer to the above questions. The fact is that if the oncological therapy including the FOLFOX 4 + bevacizumab regimen was not administered then the success of PVE in terms of liver hypertrophy was high, but the risk of tumor progression after PVE was also high. The oncological treatment was also a certain risk factor for insufficient hypertrophy of the liver parenchyma.

The drawback of our study was the number of monitored patients with CLM after PVE. Another limiting factor was the low number of patients with insufficient growth of liver parenchyma after PVE. Despite it, we assume that the acquired results are beneficial for clinical practice and our goal is to further extend the whole group of patients and to obtain a sufficient amount of data for final evaluation of this complex issue.

In conclusion, our study showed the effect of the number of CLM, liver volume and liver volume changes after PVE and concomitant oncological treatment on the final effect of PVE. Based on this finding, and after obtaining more data, we believe that early prediction of the outcome of PVE will be possible and then we will be able to choose the optimal treatment for these patients. 


\section{References}

1. Fernandez FG, Ritter J, Goodwin JW, Linehan DC, Hawkins WG, Strasberg SM. Effect of steatohepatitis associated with irinotecan or oxaliplatin pretreatment on resectability of hepatic colorectal metastases. J Am Coll Surg 2005; 200: 845-53.

2. Ellis LM, Curley SA, Grothey A. Surgical resection after down-sizing of colorectal liver metastasis in era of bevacizumab. J Clin Oncol 2005; 23: 4853-5.

3. Barbaro B, Di SC, Nuzo G. Preoperative right portal vein embolization in patients with metastatic liver disease: metastatic liver volumes after RPVE. Acta Radiol 2003; 44 . 98-102.

4. Brozek W, Bises G, Girsch T. Differentiation-dependent espression and mitogenic action of interleukin- 6 in human colon carcinoma cells: relevance for tumour progression. Eur J Cancer 2005; 41: 2347-54.

5. De Graaf W, van den Esschert JW, van Lienden KP, van Gulik TM. Induction of tumor growth after preoperative portal vein embolization: is it a real problem? Ann Surg Oncol 2009; 16: 423-30.

6. Park SH, Sung JY, Han SH, et al. Oxaliplatin, folinic acid and 5-fluorouracil (FOLFOX-4) combination chemotherapy as second-line treatment in advanced colorectal cancer patients with irinotecan failure: a Korean single-center experience. Jpn J Clin Oncol 2005; 35: 531-5.

7. Emmanoulides C, Sfakiotaki G, Androulakis N, et al. Frontline bevacizumab in combination with oxaliplatin, leucovorin and 5-fluorouracil (FOLFOX) in patients with metastatic colorectal cancer: a multicenter phase II study. BMC Cancer 2007; 7: 91-8.

8. Ryska M, Pantoflicek J, Dusek L. Surgery for liver metas tases of colorectal etiology in Czech Republic-Current National Survey. Rozhl Chir 2010; 89: 100-8.

9. Shimada H, Tanaka K, Endou I, Ichikawa Y. Treatment for colorectal liver metastases: a review. Langenbecks Arch Surg 2009; 394: 973-83.

10. Gilson N, Honoré C, De Roover A, et al. Surgical management of hepatic metastases of colorectal origin. Acta Gastroenterol Belg 2009; 72: 321-6.

11. Treska V, Skalicky T, Sutnar A, Liska V. The surgical treatment of colorectal liver metastases. Rozhl Chir 2009; 88: 69-75.

12. Neumann UP, Seehofer D, Neuhaus P. The surgical treat ment of hepatic metastases in colorectal carcinoma. Dtsch Arztbl Int 2010; 107: 335-42.

13. Farges O, Belghiti J, Kianmanesh R. Portal vein embolization before right hepatectomy: prospective clinical trial. Ann Surg 2003; 237: 208-17.

14. Ribero D, Abdalla EK, Madoff DC, Donadon M, Loyeer EM, Vauthey JN. Portal vein embolization before major hepatectomy and its effects on regeneration. Br J Surg 2007; 94: 1386-94.

15. Liu H, Zhu S. Present status and future perspectives of preoperative portal vein embolization. Am J Surg 2009; 197: 686-90

16. Gulik T, Esschert JW, Graaf W, et al. Controversies in the use of portal vein embolization. Dig Surg 2008; 25: 436-44.

17. Kokudo N, Tada K, Seki M. Proliferative activity of intrahepatic colorectal metastases after preoperative hemihepatic portal vein embolization. Hepatology 2001; 34: 267-72.

18. Hemming AW, Reed Al, Howard RJ. Preoperative portal vein embolization for extended hepatectomy. Ann Surg 2003; 237: 686-91.
19. Yokoyama Y, Nagino M, Nimura Y. Mechanisms of hepatic regeneration following portal vein embolization and partial hepatectomy: a review. World J Surg 2007; 31: 367-74.

20. Aussilhou B, Lesurtel M, Sauvanet A. Right portal vein ligation is as efficient as portal vein embolization to induce hypertrophy of the left liver remnant. J Gastrointest Surg 2008; 12: 297-303.

21. Jiang $Y$, Jahagirdar $B N$, Reinhardt RL, Schwartzm RE, Keene CD, Ortiz-Gonzales XR. Pluripotency of mesenchymal stem cells derived from adult marrow. Nature 2002; 418: 41-9.

22. Furst G, Schulte EJ, Hosch SB. Portal vein embolization and autologous CD 133+ bone marrow stem cells for liver regeneration: initial experience. Radiology 2007; 234: 171-9.

23. Komori K, Nagino M, Nimura Y. Hepatocyte morphology and kinetics after portal vein embolization. Br J Surg 2006; 93: 745-51.

24. Furrer K, Tian Y, Pfammatter T, et al. Selective portal vein embolization and ligation trigger different regenerative responses in the rat liver. Hepatology 2008; 47: 1615-23.

25. Kokudo N, Tada K, Seki M, et al. Proliferative activity of intrahepatic colorectal metastases after preoperative hemihepatic portal vein embolization. Hepatology 2001; 34: 267-72

26. Ogata S, Belghiti J, Farges O, Varma D, Silbert A, Vilgrain V. Sequential arterial and portal vein embolizations before right hepatectomy in patients with cirrhosis and hepatocellular carcinoma. Br J Surg 2006; 93: 1091-8.

27. Hayashi H, Beppu T, Sugita H, et al. Serum HGF and TGFbeta1 levels after right portal vein embolization. Hepatology Res 2010; 40: 311-7.

28. Selzner N, Pestalozzi BC, Kadry Z, Selzner M, Wildermuth S, Clavien PA. Downstaging colorectal liver metastases by concomitant unilateral portal vein ligation and selective intraarterial chemotherapy. Br J Surg 2004; 93: 587-92.

29. Hayashi S, Baba Y, Ueno K. Acceleration of primary liver tumor growth rate in an embolized hepatic lobe after portal vein embolization. Acta Radiol 2007; 48: 721-7.

30. Jaeck D, Bachellier P, Nakano H. One or two-stage hepatectomy combined with portal vein embolization for initially nonresectable colorectal liver metastases. Am J Surg 2003; 185: 221-9.

31. Beal IK, Anthony S, Papadopoulou A. Portal vein embolisation prior to hepatic resection for colorectal liver metastases and the effects of periprocedure chemotherapy. $\mathrm{Br}$ J Radiol 2006; 79: 473-8.

32. Goere D, Farges O, Leporrier J. Chemotherapy does not impact hypertrophy of the left liver after right portal vein obstruction. J Gastrointest Surg 2006; 10: 365-70.

33. Tanaka K, Kumamoto T, Matsuyama R, Takeda K, Nagano Y, Endo I. Influence of chemotherapy on liver regeneration induced by portal vein embolization or first hepatectomy of a staged procedure for colorectal liver metastases. J Gastrointest Surg 2010; 14: 359-68.

34. Fehér J, Németh E, Lengyel G. Non-alcoholic steatohepatitis (NASH). Arch Med Sci 2005; 1: 37-47.

35. Treska V, Skalicky T, Sutnar A, et al. Portal vein branch embolization in patients with primary inoperable liver tumors. Rozhl Chir 2010; 9: 456-60.

36. Boxberger F, Albrecht H, Konturek $\mathrm{P}$, et al. Neoadjuvant treatment with weekly high-dose 5 -fluorouracil as a $24 \mathrm{~h}$ infusion, folinic acid and biweekly oxaliplatin in patients with primary resectable liver metastases of colorectal cancer: long-term results of a phase II trial. Med Sci Monit 2010; 16: CR 49-55. 
37. Stec R, Grala B, Maczewski M, Bodnar L, Zegadlo A, Szczylik C. Combination of monoclonal antibody (Bevacizum$\mathrm{ab})$ and chemotherapy (FOLFIRI) in paliative treatment of metastatic adenocarcinoma of the small bowel (SBC): case report and review of the literature. Arch Med Sci 2009; 5: 647-51.

38. Belghiti J, Benhaim L. Portal vein occlusion prior to extensit resection in colorectal liver metastasis: a necessity rather than an option. Ann Surg Oncol 2009; 16: 1098-9.

39. Wicherts DA, de Haas RJ, Andreani P, et al. Impact of portal vein embolization on long-term survival of patients with primarily unresectable colorectal liver metastases. Br J Surg 2010; 97: 240-50.

40. Jaeck D, Oussoultzoglou E, Rosso E. A two-stage hepatectomy procedure combined with portal vein embolization to achieve curative resection for initially unresectable multiple and bilobar colorectal liver metastases. Ann Surg 2004; 240: 1037-49. 